\title{
Quality Analysis for Different Video Formats by Using Video Watermarking
}

\author{
Sharanjeet Kaur ${ }^{\mathrm{a}}$, Ramandeep Kaur ${ }^{\mathrm{b}}$
}

${ }^{a}$ Research Scholar, Department of Computer Engineering, CT Group of Institutions, Jalandhar, 144001, India

${ }^{b}$ Research Scholar, Department of Computer Engineering, CT Group of Institutions, Jalandhar, 144001, India

\begin{abstract}
In this paper, our main motive to compare different video formats and also analyze what will be the effect on quality when we embed same watermark in different type of formats such as .avi (colored and uncompressed video format), mp4(colored and compressed video format) \& grey color image using discrete wavelet transform \& singular value decompositions techniques. In research work, HH bands are used to embed the watermark into video because this band are less sensitive to human eyes and not easily detected or captured by human visual system. It also helps to improve the quality of video. The analysis is done on the basis of quality metrics such as PSNR, BER \& MSE for different format video clips.
\end{abstract}

Index Terms: Video Watermarking, Singular Value Decomposition, Discrete Wavelet Transform, Security \& Copyright Protection.

(C) 2016 Published by MECS Publisher. Selection and/or peer review under responsibility of the Research Association of Modern Education and Computer Science.

\section{Introduction}

Recently, video watermarking has become very popular research area and this technique provides a security and copyright protection against piracy, fraud and misused of data. The watermark is embed into video in a way which can't be noticed by human eyes and transfer it over the network without any loss of quality of video. Watermarking is used since the ancient time to protect the private information by hackers by using different types such as text, audio, video and image watermarking. The aim of watermarking is provide the identity to real owner of the video. Watermarking is different from cryptography and steganography. Cryptography is basically used to scramble a message and after decrypted the message doesn't provide security. But in case of watermarking is highly secured and robust against attacks. In watermarking the information is secured due to the company logo or captions embed inside the video which can't be easily damaged or removed by hackers. It

*Corresponding author

E-mail Address: a sharanmarock6@gmail.com,b raman177rk@gmail.com 
also helps to protect the media files by embedding logo inside the files and provide authentication. On other hand steganography is used to hide the message inside the files. In case of robustness both are measured on different aspects steganography concerned with detection of the message and watermarking is concerned with quality of video and remove by a piracy. Video watermarking work in two phases, embedding and extraction phase. Embedding phase is basically done on sender side. In this phase, we basically inserted the logo or image inside video. On other hand, extraction process is done on receiver side and it is reverse process of embedding process and it basically used to extract the watermark logo or image from the video. Embedding algorithm is basically divides into two domain spatially domain and frequency domain. In spatially domain the watermarking algorithms are directly loaded into the real image. The example of spatially domain is LSB (least significant bit). In frequency domain, the values of the certain frequencies are modified from their original values. It is also called transform domain. Examples of frequency domain are DCT (discrete cosine transform), DWT (discrete wavelet transform) and DFT (discrete Fourier transform). Best watermarking always hold these characteristics:

- Robustness: If, inserted watermark should be present inside the video after various attacks then it indicates it highly robust and your information is safe.

- Security: It is the major tool to secure the digital objects from hackers. It can be enhanced by using various encryption methods and algorithms.

- Imperceptibility: This term is also known as invisible or transparent. It indicates that no difference is detected before and after inserted watermark inside into video. It is basically deal with two terms i.e. quality and fidelity.

- Data Payload Size \& Capacity: How much data or number of bits is inserted in a host signal is called as data payload size. The total amount of information is embedded inside the video is called as capacity.

This paper defines the concept of watermarking by using a hybrid approach for video watermarking by using Discrete Wavelet Transformation (DWT) \& Singular Value decomposition techniques to improve the robustness, quality, security \& data payload size. It has been also carried out a comparative analysis using different video formats to analyze the quality of watermarked video. The results have been taken out practically by using MATLAB toolbox for simulation. The whole research paper is arranged into various sections; Section 2 describes the related work, Section 3 presents the proposed methodology, Section 4 explains the embedding and extraction algorithms, Section 5 consist of simulated results \& discussion, and Section 6 discuss the conclusion and future scope of the proposed work.

\section{Related Work}

The main aim of video watermarking is to provide a copyright protection and authentication to the digital object and solve the issues of piracy, duplicity and fraud over the internet. To solve this issue by using various techniques and algorithms have been proposed to overcome the effects of attacks on video and also maintain the quality of video.

In year 2015 Ref. [14] author has purposed a fusion technique with the help of encryption method by using discrete wavelet transform. DWT technique is used to insert the watermark inside the video and encryption scan method is used to improve the security by rearranging the pixels value. At the extraction time, there is no difference detect between the original \& watermarked video. This paper provides a highly robustness, security and imperceptibility of video. Limitation of this paper is scan encryption method takes a lot of time during rearranging the pixels value.

In year 2014 Ref. [15] this paper by using DWT technique, author successfully performed image watermarking on video frames. In this technique, we divide the image into RGB components and apply DWT watermarking on every single frame of the video. This technique enhances the security and gives permission to recover the extract RGB image without any damage or distortion. It also helps to improve the value of PSNR 
and MSE value and increase the visual quality of the video. Limitation of this paper is DWT cost of computing is higher applying DWT watermarking on every single frame of the target video. It also causes a noise near video frames.

In year 2013 Ref [18] author has select an appropriate embedding image blocks \& these same blocks are used for extracting process by using SVD \& DWT techniques. This scheme helps to provide a copy right protection to the real owner of the digital content and also provide good quality of video. In Ref. [6] In this paper QR code prepared to watermark via robust video watermarking scheme based on the SVD and DWT. SVD is an attractive algebric transform watermarking. SVD is applied to the cover I- frame. The diagonal value is fused with logo or watermark. DWT is applied on SVD and QR code image. This method can achieve the acceptable imperceptibility and robustness in video processing. The limitation of this paper is QR code have some security issue because before scanning the scanner can really never know where the code is going to lead them so, QR code also have a synchronization problem.

In Ref. [9] this paper, author present a strong video watermarking algorithm for RGB .avi video. Author used two major techniques of video watermarking i.e. discrete wavelet transform \& Singular value decomposition. For embedding process, firstly apply a scene change method to video and after that watermark is embedded into singular values of $3^{\text {rd }}$ level LL band of video frames. This paper provides high imperceptibility of video and robustness against various attacks. The algorithm robust and shows an improvement in quality of video. The limitation of this paper is scene change method is not so secure and it's easily cracks by hackers.

In year 2012 Ref. [11] author proposed a great algorithm to stop the misused of data and provide copyright protection by using discrete wavelet transform with blind scene algorithm. Spread spectrum technique is used to embed the watermark into selected wavelet coefficients. Original video is not needed for extraction process because of blind video algorithm. The resultant output provides a transparency and robustness. Limitation of spread spectrum technique is codes need to be exactly orthogonal.

\section{Proposed Methodology}

The proposed methodology for a video watermarking by using discrete wavelet transform \& singular value decomposition based on different video formats. Our main goal is to analyze the impact of quality of watermarked videos by using different video formats when we embed same watermark image inside cover video by following same algorithm based video watermarking. The following are the main steps for proposed methodology.

- Video Selection Process: This is a first step of methodology in which we select video format in which we want to embed a watermark. There is different type videos of different video formats are available such as .avi \& .mpg \& .mp4 etc. we can choose any one at a time.

- Frame Extraction: Extract 10 random frames from video by using random frame selection algorithm on the basis of symmetric key.

- Embedding Process: embed the same watermark image behind different videos such as .avi, .mp4 and other one by one using second level of DWT \& SVD and generate watermark video for each selected video formats.

- Extraction Process: To extract the watermark from the watermarked video, perform reverse process of embedding algorithm using symmetric key \& obtain the original video without any loss of quality.

- Attacks: Apply various operations on the video to check the robustness of different format of video.

- Comparison: Calculate MSE, PSNR, BER and SSIM for each video after embedding watermark in different format videos to analyze the affect of quality.

\section{Algorithm}

Algorithms which we used this work is explained below: 


\subsection{Embedding Algorithm}

The embedding process is used to inserted watermark inside media file. It is carried out at sender side. The following steps and flow chart are followed for proposed methodology:

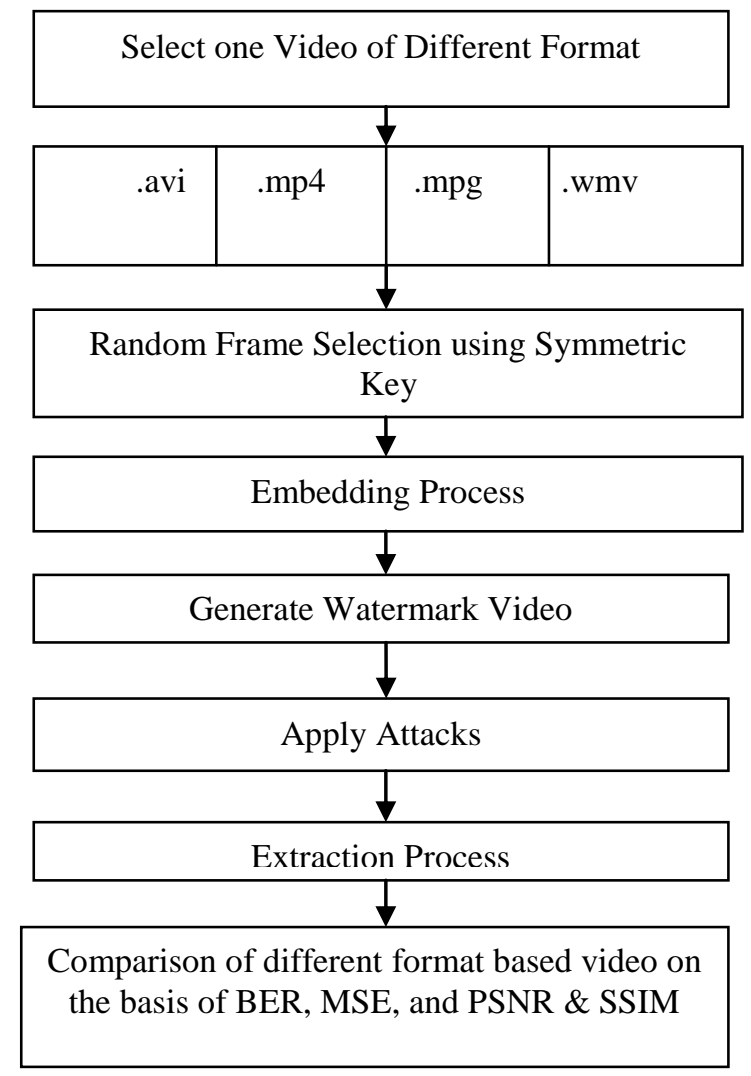

Fig.1. Flow Chart of Proposed Methodology

- Select one video of any format either .avi/.mp4/.mpg or any other format video. Suppose, we are selecting .mpg format based video file and calculate total its frame rate as shown in Fig.1.

- Select 10 random frames from video frames using symmetric key.

- Select watermark image and 10 digit symmetric key.

- Hide symmetric key \& selected frame addresses in first frame of video.

- $\quad$ Now, embed watermark using $2^{\text {nd }}$ level DWT \& SVD generate watermark video file.

- Repeat step 1 to 5 for selecting next format based video i.e. mp4, .wov or avi video and embed same watermark image using same algorithm steps for embedding data inside video file.

Analyze effect of quality of different format based video files on basis of PSNR, MSE, BER and SSIM and analyze affect of quality.

\subsection{Extraction Algorithm}


The extraction process is a reverse process of embedding algorithm used to watermark image from media file and it is carried out at receiver side. The extraction steps are as follow:

- Select watermarked video and enter same 10 digit symmetric key at receiver side to extract watermark.

- Extract secret key, selected frame addresses from $1^{\text {st }}$ frame of watermarked video.

- Extract watermark by using reverse process of embedding algorithm.

- To obtain the original video without any loss of quality or damage of video.

- Exit.

\section{Experimental Results}

The proposed algorithm simulation is done on MATLAB 7.0 version 2014a. The experimental results show in given below:

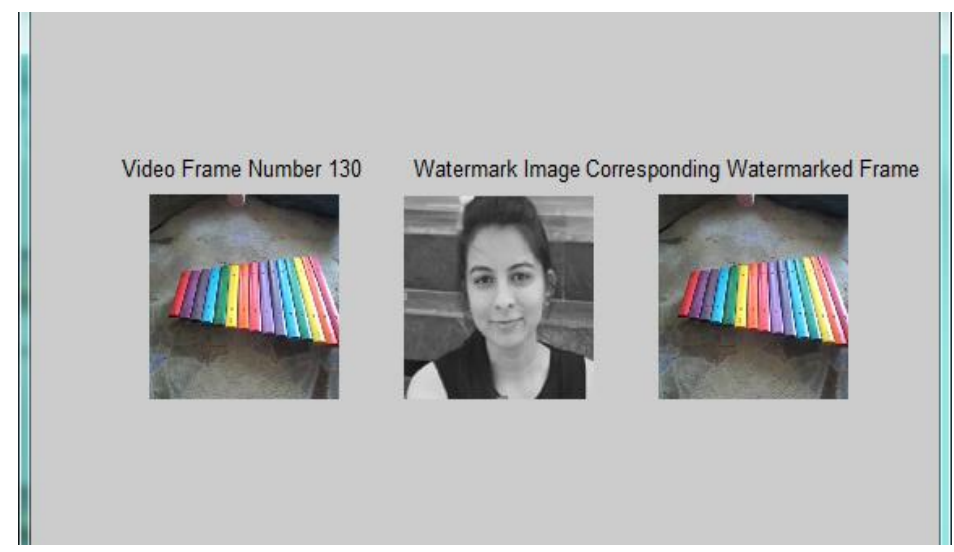

Fig.2. Showing video frame, watermark image \& watermarked frame for .mpg video

Table 1. Testing Results for .mpg video format

\begin{tabular}{ccc}
\hline $\begin{array}{c}\text { Video Clip } \\
\text { Format }\end{array}$ & $\begin{array}{c}\text { Parameters } \\
\text { Name }\end{array}$ & $\begin{array}{c}\text { Average } \\
\text { Values }\end{array}$ \\
\hline \multirow{3}{*}{ Xylophone } & BER & 0.012 \\
& PSNR & 78.20 \\
& MSE & 0.000 \\
& SSIM & 1 \\
\hline
\end{tabular}




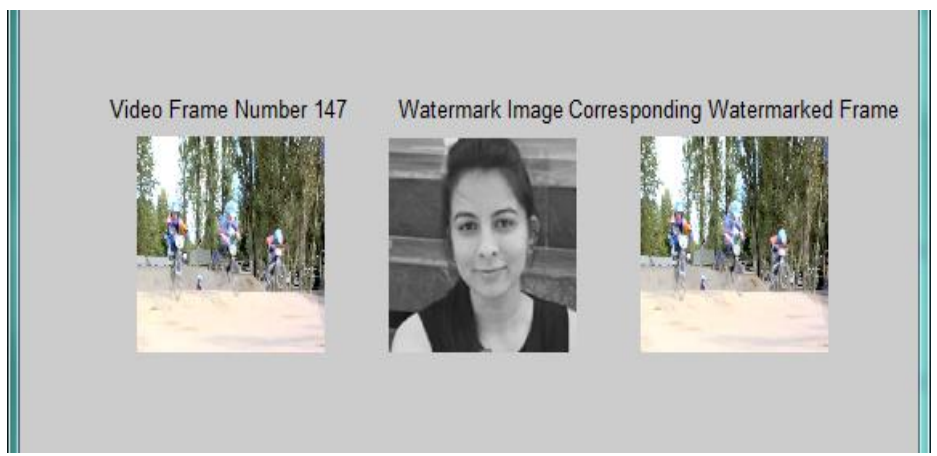

Fig.3. Showing video frame, watermark image \& watermarked frame for .mp4 video

Table 2. Testing Results for .mp4 video format

\begin{tabular}{lcc}
\hline $\begin{array}{l}\text { Video Clip } \\
\text { Format }\end{array}$ & $\begin{array}{c}\text { Parameters } \\
\text { Name }\end{array}$ & $\begin{array}{c}\text { Average } \\
\text { Values }\end{array}$ \\
\hline \multirow{2}{*}{ PP4 } & BER & 0.012 \\
& PSNR & 78.03 \\
& MSE & 0.001 \\
& SSIM & 1 \\
\hline
\end{tabular}

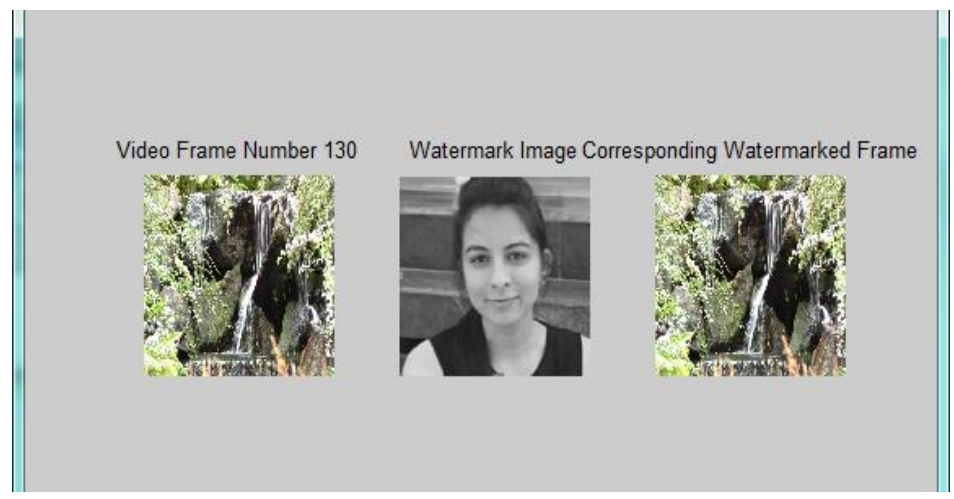

Fig.4. Showing video frame, watermark image \& watermarked frame for .wmv video

Table 3. Testing Results for .wmv video format

\begin{tabular}{ccc}
\hline $\begin{array}{c}\text { Video Clip } \\
\text { Format }\end{array}$ & $\begin{array}{c}\text { Parameters } \\
\text { Name }\end{array}$ & $\begin{array}{c}\text { Average } \\
\text { Values }\end{array}$ \\
\hline \multirow{3}{*}{ winsat.wmv } & BER & 0.012 \\
& PSNR & 79.12 \\
& MSE & 0.000 \\
& SSIM & 1 \\
\hline
\end{tabular}




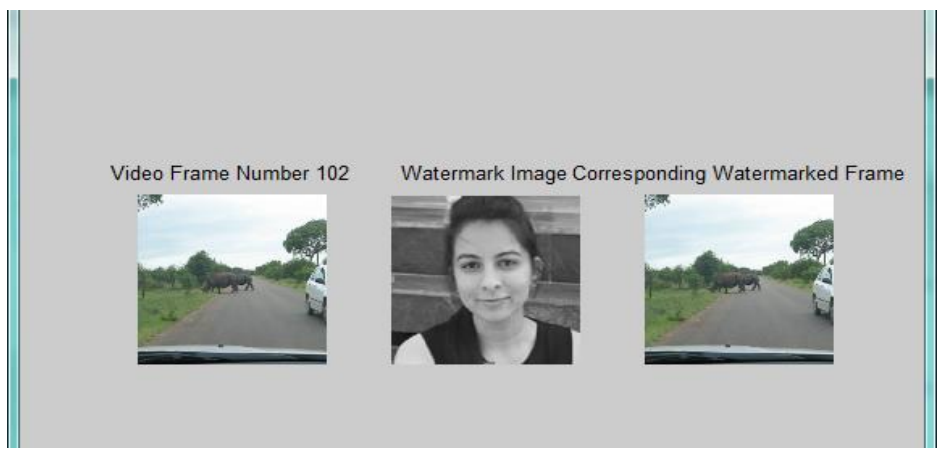

Fig.5. Showing video frame, watermark image \& watermarked frame for .avi video

Table 4. Testing Results for avi video format

\begin{tabular}{ccc}
\hline $\begin{array}{c}\text { Video Clip } \\
\text { Format }\end{array}$ & $\begin{array}{c}\text { Parameters } \\
\text { Name }\end{array}$ & $\begin{array}{c}\text { Average } \\
\text { Values }\end{array}$ \\
\hline \multirow{2}{*}{ rhinos.avi } & BER & 0.012 \\
& PSNR & 72.77 \\
& MSE & 0.001 \\
& SSIM & 0.9999 \\
\hline
\end{tabular}

\subsection{Attacks on different format of video}

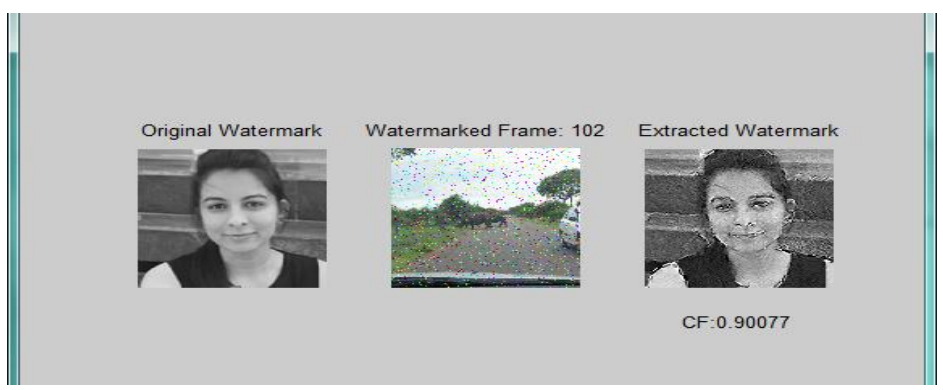

Fig.6. Salt \& Pepper Attack Apply on Rhinos.avi video

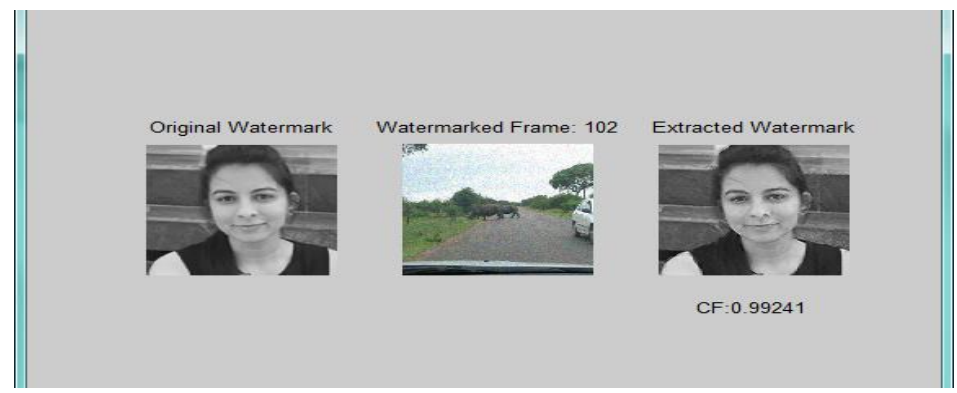

Fig.7. Poisson Noise Attack Apply on Rhinos.avi video 


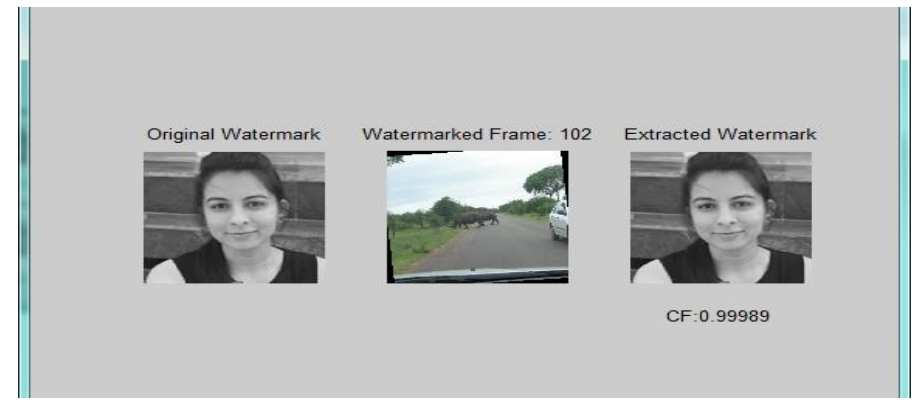

Fig.8. Rotational Attack Apply on Rhinos.avi video

Table 5. Extraction results of Attacks for .avi video

\begin{tabular}{clll}
\hline $\begin{array}{c}\text { Video Clip } \\
\text { Format }\end{array}$ & $\begin{array}{c}\text { Attacks } \\
\text { Names }\end{array}$ & \multicolumn{2}{l}{ Parameter Values } \\
\hline \multirow{2}{*}{ rhinos.avi } & Salt \& Pepper & CF \\
& Poisson Noise & 36.29 & 0.9 \\
& Rotational Attack & 54.35 & 0.9 \\
\hline
\end{tabular}

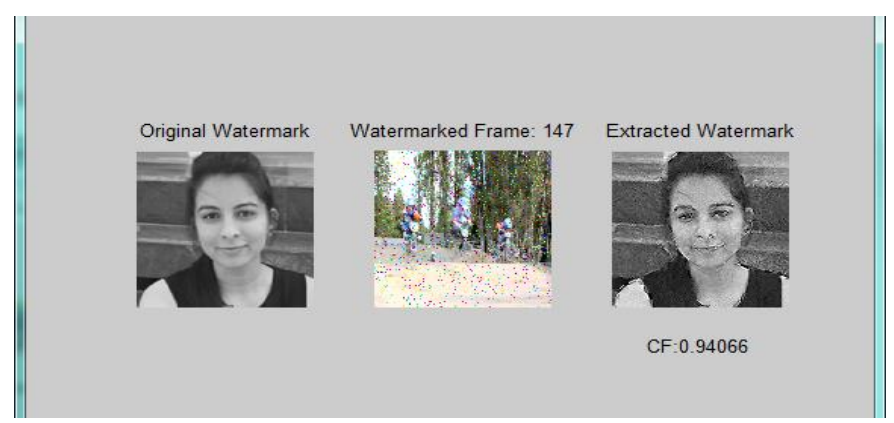

Fig.9. Salt \& Pepper Noise Attack Apply on .mp4 Video

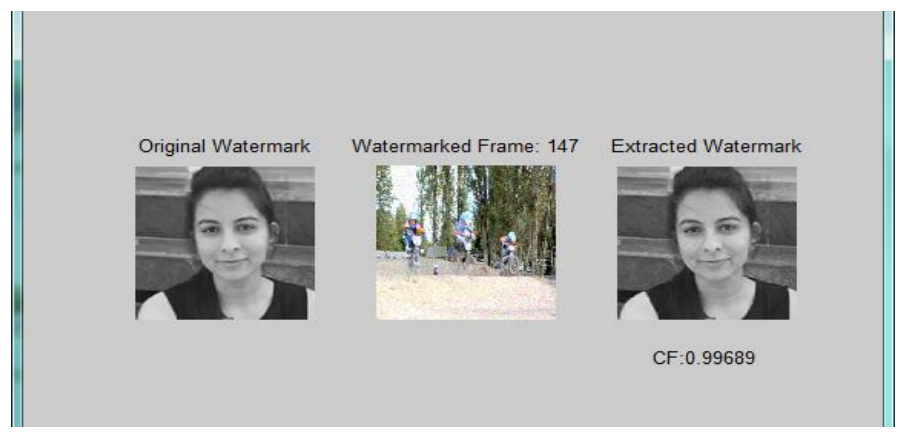

Fig.10. Poisson Noise Attack Apply on.mp4 Video 
Table 6. Extraction results of Attacks for .mp4 video

\begin{tabular}{lccc}
\hline $\begin{array}{c}\text { Video Clip } \\
\text { Format }\end{array}$ & $\begin{array}{c}\text { Attacks } \\
\text { Names }\end{array}$ & \multicolumn{2}{c}{ Parameter Values } \\
& & PSNR & CF \\
\hline .mp4 & Salt \& Pepper & 30.56 & 0.9 \\
& Poisson Noise & 37.34 & 0.9 \\
& & & \\
\hline
\end{tabular}

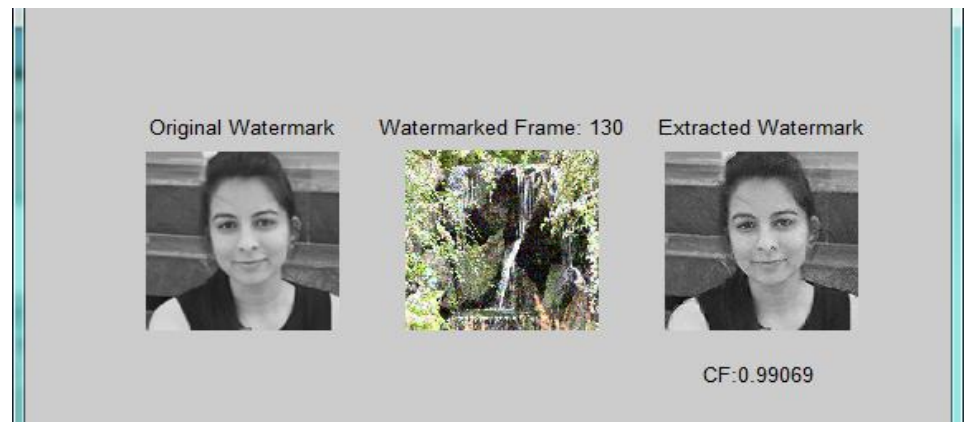

Fig.11. Gaussian Noise Attack Apply on .wmv video

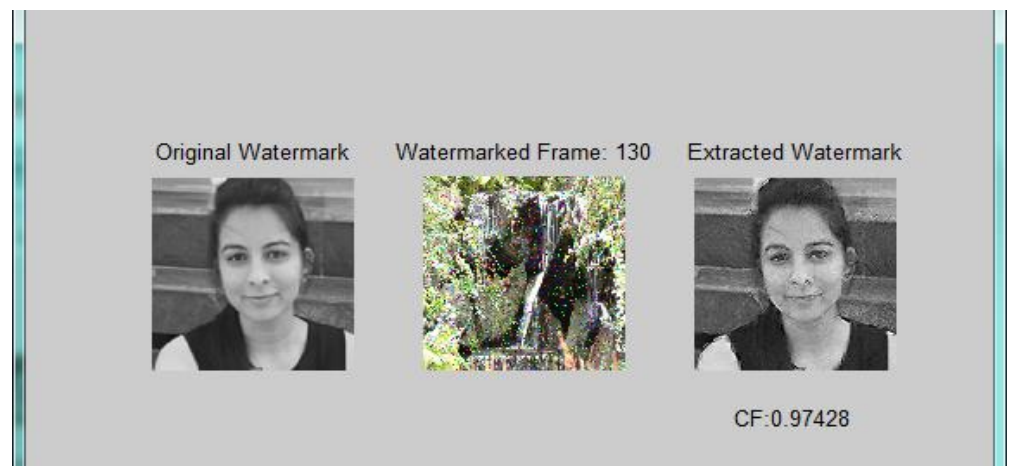

Fig.12. Salt \& Pepper Attack Apply on .wmv video

Table 7. Extraction results of Attacks for .wmv video

\begin{tabular}{cccc}
\hline $\begin{array}{c}\text { Video Clip } \\
\text { Format }\end{array}$ & $\begin{array}{c}\text { Attacks } \\
\text { Names }\end{array}$ & \multicolumn{2}{c}{ Parameter Values } \\
\hline Winsat.wmv & Salt \& Pepper & 31.40 & CF \\
& Gaussian Noise & 33.53 & 0.9 \\
\hline
\end{tabular}




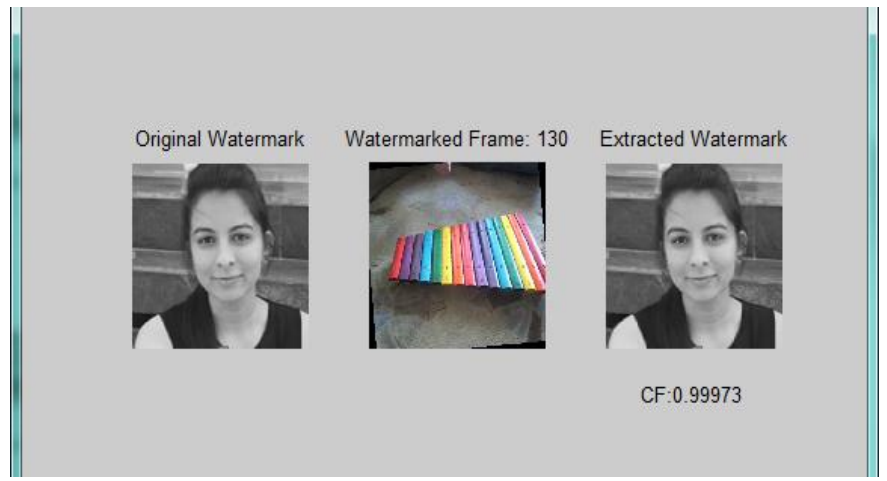

Fig.13. Rotational Attack apply on .mpg video

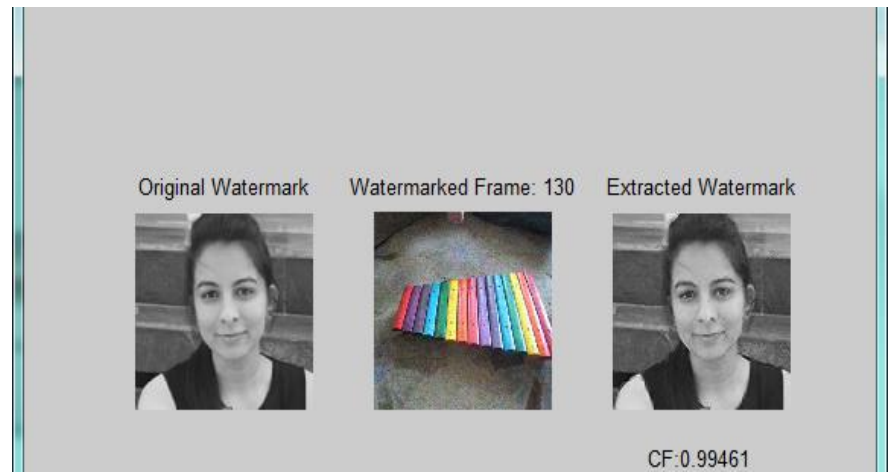

Fig.14. Poisson Attack apply on .mpg video

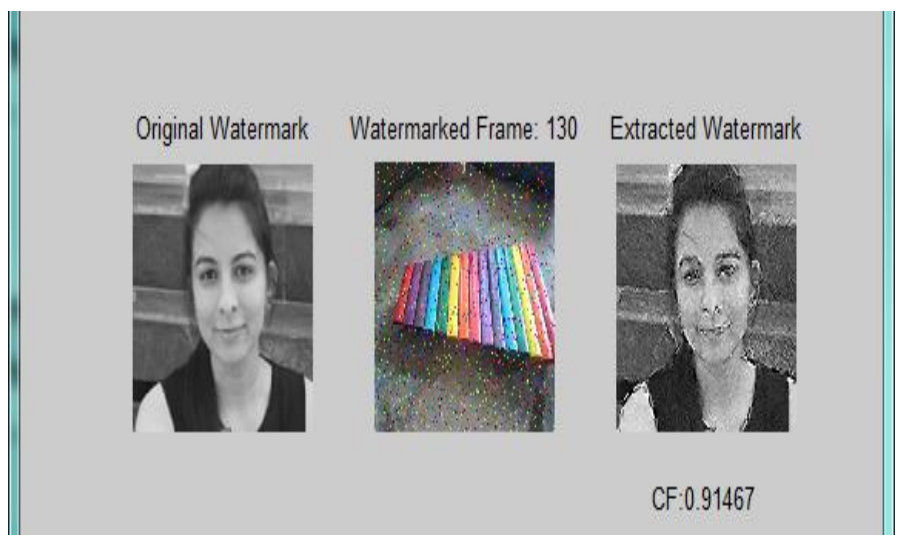

Fig.15. Salt \& Pepper Noise Attack on .mpg Video 
Table 8. Extraction results of Attacks for .mpg video

\begin{tabular}{clcc}
\hline $\begin{array}{l}\text { Video Clip } \\
\text { Format }\end{array}$ & $\begin{array}{l}\text { Attacks } \\
\text { Names }\end{array}$ & \multicolumn{2}{c}{ Parameter Values } \\
& PSNR & CF \\
\hline \multirow{2}{*}{ Xylophone.mpg Salt \& Pepper } & 30.12 & 0.9 \\
& Poisson Noise & 37.79 & 0.9 \\
& Rotational Attack & 50.32 & 0.9 \\
\hline
\end{tabular}

\section{Conclusions\& Future Scope}

The testing analysis is conclude that proposed algorithm is highly resist against salt \& pepper attack, Gaussian noise, Poisson noise and geometrical attack such as rotational attack. Therefore the proposed algorithm is provides highly secure and good quality of video by using second level of DWT and SVD. In future, new research scholar can do their research work by following the same algorithm for audio steganography and by using different audio formats such as .mp3 and .wave etc.

\section{Acknowledgement}

We wish to thank parents, teachers, supervisors and friends for their great support and help during the making of this research paper. We also thank all researchers whose previous work gives us a way to extend and complete research work.

\section{Appendix A. Abbreviations}

\begin{tabular}{|l|l|}
\hline AVI & Audio and Video Interleaved \\
\hline MPEG & Motion Picture Expert Group \\
\hline MP4 & MPEG Type-4 \\
\hline WMV & Window Media Video Format \\
\hline MP4 & MPEG Layer 4 Type Format \\
\hline PNG & Portable Network Graphics \\
\hline DWT & Discrete Wavelet Transform \\
\hline SVD & Singular Value Decomposition \\
\hline
\end{tabular}

\section{References}

[1] Sharanjeet kaur, Pooja \& Varsha, "Copyright Protection of data by using Video Watermarking" International Journal of Computer Applications (IJCA), Vol.119, No.17, June 2015, pp: 14-18.

[2] Sharanjeet kaur, Pooja, " 3 rd level of DWT\& SVD based Video Watermarking" International Journal of Science and Research (IJSR), Vol.4, Issue 11, November 2015, pp: 1483-1487.

[3] Amrinder Singh, Sukhjit Singh "A Robust Video Watermark Embedding and extraction Technique Based on Random Frame Selection” IJCA Vol. 2, NO.2, Feb 2014. 
[4] Tanima Dutta, Arijit Sur, Sukumar Nandi, "A Robust Compressed Domain video watermark P-frame with Controlled Bit Rate Increase”, IEEE 2013.

[5] Hamid Shojanazeri, Wan Azizun Wan Adnan, Sharifah Mumtadzah Syed Ahmad, M. Iqbal Saripan,"Analysis of Watermark Techniques in Video", IEEE 2011. pp 486-492.

[6] G.Prabakaran, R.Bhavani, M.Ramesh," A Robust QR- Code Video Watermarking Scheme Based on SVD and DWT Composite Domain", IEEE International Conference On Pattern Recognition, Informatics And Mobile Engineering (Prime), February 2013. pp 251-257.

[7] Anita Jadhav, Megha Kolhekar," Digital Watermarking in Video for Copy Right Protection" IEEE International Conference on Electronic Systems, Signal Processing and Computing Technologies, 2014. pp 140-144.

[8] Harshita Rawat, Ashwani Kumar, Satendra Kumar," Robust Digital Image Watermarking Scheme for Copyright Protection”, Volume 75, No.18, August 2013.pp 27-32.

[9] Bhavna Goel, Charu Agarwal," An Optimized Un-compressed Video Watermarking Scheme based on SVD and DWT", IEEE 2013, pp 307- 312.

[10] Osama S.Fargallah," Efficient video watermarking based on singular value decomposition in the discrete wavelet transform domain", Elsevier 2012, pp 189- 196.

[11] Majid Masoumi ,Shervin Amiri,"A blind scene-based watermarking for video copyright protection", Elsevier 2012, pp 528 - 535.

[12] Angshumi sarma,Amrita Ganguly," An Entropy based Video Watermarking Scheme", International Journal of Computer Applications, volume 50, no.7, July 2012, pp 33 -37.

[13] Jonathen Blake, Shahram Latifi,"Digital Watermarking Security" Defence Science Journal, Volume 61, NO.5, September 2011, pp 408-414.

[14] Monika Sharma, Archana Tiwari, "A Fusion Technique of Video Watermarking in Wavelet Domain and Encryption Method for Video Authentication", International Journal of Science and Research (IJSR), Vol.115, Issue 1, November 2015, pp: 30-34.

[15] Saket Kumar, Ashutosh Gupta Ankur Chandwani, Gaurav Yadav, Rashmi Swarnkar," RGB Image Watermarking on Video Frames using DWT”, IEEE 2015, pp: 675-680.

[16] Gaurav Bhatnagar, Balasubramanian Raman," Wavelet packet transform-based robust video watermarking technique" Indian Academy of Science, volume 37, no.3, June 2012, pp371-388

[17] Gopika V Mane, G.G. Chiddarwar," Review Paper on Video Watermarking Techniques" International journal of Scientific and Research Publication, volume 3, no.4, April 2013. Pp: 1-5.

[18] Rajesh Mehta, Navin Rajpal," A Hybrid Semi Blind Gray Scale Image Watermarking Algorithm Based on DWT-SVD using Human Visual System Model”, IEEE 2013. pp 163-168.

[19] Ghassan.N Mohammed, Azman Yasin, Akram M.Zeki," Digital Image Watermarking, Analysis of Current Methods", IEEE International conference on Advanced Computer Science Application and Technologies, 2013. pp 324-329.

[20] Nilesh Kumar Dubey, Shishir Kumar," A Review of Watermarking Application in Digital Cinema for Piracy Deterrence", IEEE International Conference on Communication Systems and Networking Technologies, 2014. Pp 626-630.

[21] Min Liu," Study of Digital Image Watermarking", IEEE International Conference on Computer Science and Electronics Engineering, 2012. pp 77-80.

[22] Manekandan.GRS, Franklin Rajkumar.Vs," A Robust Watermarking Scheme for Digital Video Sequence using Entropy and Hadamard Transformation Technique", International Journal of Computer Application, volume 41, NO.18, March 2012. pp 24-31.

[23] Saeed Rastegar, Fateme Namazi, Khashayar Yaghmaie, Amir Aliabadian ," Hybrid watermarking algorithm based on Singular value Decomposition and Radon Transform", Elsevier 2010. pp 658-663.

[24] Radu O. Preda, Dragos N. Vizireanu', A robust digital watermarking scheme for video copyright protection in the wavelet domain", Elsevier 2010. pp 1720-1726. 
[25] Hamid Shojanazeri, Wan Azizun Wan Adnan, Sharifah Mumtadzah Syed Ahmad, "Video Watermarking Techniques for Copyright protection and Content Authentication", International Journal of Computer Information Systems and Industrial Management Applications, volume 5, 2013, pp 652 - 660.

\section{Authors' Profiles}

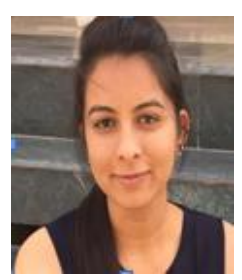

Sharanjeet Kaur born on January 16, 1992 lives in Punjab, India. She has completed his B-TECH from college of engineering \& management, kapurthala in 2013 and pursing MECH from CT group of institute, jalandhar. She has attended various seminar and workshops related research work. Her interest of area is Digital Image Processing, networking \& information Security \& multimedia.

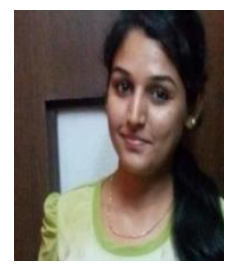

Ramandeep Kaur born on February 22, 1992 lives in Punjab. She has completed his BTECH from college of engineering \& management, kapurthala in 2013 and pursing M-ECH from CT group of institute, jalandhar. She has attended various seminar and workshops related research work. Her interest of area is Digital Image Processing, multimedia \& networking \& information Security.

How to cite this paper: Sharanjeet Kaur, Ramandeep Kaur,"Quality Analysis for Different Video Formats by Using Video Watermarking", International Journal of Engineering and Manufacturing(IJEM), Vol.6, No.4, pp.9-21, 2016.DOI: 10.5815/ijem.2016.04.02 